\title{
Investigation into the Performance of a MIMO System Equipped with ULA or UCA Antennas: BER, Capacity and Channel Estimation
}

\author{
Xia LIU ${ }^{1}$, Marek E. BIALKOWSKI ${ }^{2}$, Feng WANG ${ }^{1}$, Konstanty BIALKOWSKI ${ }^{3}$ \\ ${ }^{1}$ Student Member IEEE, School of ITEE, The University of Queensland, Brisbane, Australia \\ ${ }^{2}$ Fellow IEEE, School of ITEE, The University of Queensland, Brisbane, Australia \\ ${ }^{3}$ Member IEEE, National ICT Australia-Queensland Lab, Brisbane, Australia \\ Email: \{xialiu, meb, fwang, ksb\}@itee.uq.edu.au \\ Received June 15, 2009; revised July 27, 2009; accepted August 30, 2009
}

\begin{abstract}
This paper reports on investigations into the performance of a Multiple Input Multiple Output (MIMO) wireless communication system employing a uniform linear array (ULA) at the transmitter and either a uniform linear array (ULA) or a uniform circular array (UCA) antenna at the receiver. The transmitter is assumed to be surrounded by scattering objects while the receiver is postulated to be free from scattering objects. The Laplacian distribution of angle of arrival (AOA) of a signal reaching the receiver is postulated. The performance of bit error rate (BER), capacity and channel estimation for a MIMO system are evaluated for the two cases that the receiver is equipped with ULA or with UCA antennas.
\end{abstract}

Keywords: MIMO, BER, BPSK, FSK, Channel Capacity, EDOF, Channel Estimation, ULA, UCA, Spatial Correlation

\section{Introduction}

In recent years, there has been a growing interest in the communication research community in the signal transmission technique employing multiple element antennas both at the transmitter and receiver sides of a wireless communication system. The reason is that it can significantly improve the transmission quality in terms of data throughput (capacity) and coverage area without the need for extra operational frequency bandwidth. Known as the multiple-input multiple-output (MIMO) technique, it is one of the promising techniques for the next generation of mobile communications. For its physical implementation, the MIMO technique frequently assumes uniform linear arrays (ULA) at both the transmitter and receiver ends of a wireless communication system. However, to obtain operation with larger angular views, uniform circular arrays (UCA) and their similarities such as triangular, square, pentagonal or hexagonal arrays are also considered. It can be expected that different configurations of antenna arrays will result in different spatial correlations of transmitted/received signals and thus they will influence channel properties between transmit- ter and receiver in a different way. It is well known that MIMO channel capacity performance is based on the properties or channel matrix. According to [1] and [2], MIMO system BER performance and training-based channel estimation performance are determined by the channel correlation matrix which is affected by channel properties. These, in turn, the MIMO systems employ ULA or UCA receiver will affect the bit error rate (BER), channel estimation and the MIMO system capacity distinctly.

In this paper, calculations of the MIMO system BER are performed for two modulation schemes, BPSK and FSK for both noncoherent and coherent cases. For channel estimation the SLS and MMSE estimation methods are considered. In the undertaken investigations it is assumed that the receiver employs either ULA or UCA antennas while the transmitter uses only ULA. Also assumed is that the transmitter is surrounded by scattering objects while the receiver is free from scatterers. To determine the antenna array spatial correlation pattern, a Laplacian distribution for the angle of arrival (AOA), which provides a good agreement with the measured data [3], is postulated. 


\section{System Model}

\subsection{System Configuration and Spatial Correlations}

Figure 1 shows the configuration of the investigated MIMO system. The case of $4 \times 4$ MIMO is considered.

The transmitter is assumed to be equipped with a ULA antenna surrounded by scattering objects that are uniformly distributed in a circle. Antenna elements in the array have an omnidirectional radiation pattern in the azimuth plane. The considered case represents a mobile station operating close to the ground where many surrounding obstacles are expected. In turn, the receiver is assumed to be equipped with either ULA or UCA of omnidirectional antenna elements free from any surrounding obstacles. This configuration can represent a base station with antennas located high above the ground where there are no scattering objects.

In Figure 1, $\theta$ stands for the central AOA which is determined by the physical position of dominant scatterers with respect to the receiving antenna array. Assuming that the AOA follows the Laplacian distribution, the mathematical expressions for the real and imaginary parts of spatial correlation between the $m$-th and $n$-th antenna for the case of UCA receiving antenna are given as [3]:

$$
\begin{gathered}
\operatorname{Re}\{\operatorname{Rr}(m, n)\}=J_{0}\left(Z_{c}\right)+ \\
2 \sum_{k=1}^{\infty} \frac{a^{2}\left(1-e^{-a \pi}\right)}{a^{2}+4 k^{2}} J_{2 k}\left(Z_{c}\right) \cos [2 k(\theta+\alpha)] \\
\operatorname{Im}\{\operatorname{Rr}(m, n)\}=4 C_{l} \sum_{k=0}^{\infty} \frac{a^{2}\left(1-e^{-a \pi}\right)}{a^{2}+(2 k+1)^{2}} \\
\cdot J_{2 k+1}\left(Z_{c}\right) \sin [(2 k+1)(\theta+\alpha)]
\end{gathered}
$$

where $C_{l}$ is a normalizing constant given as [3]:

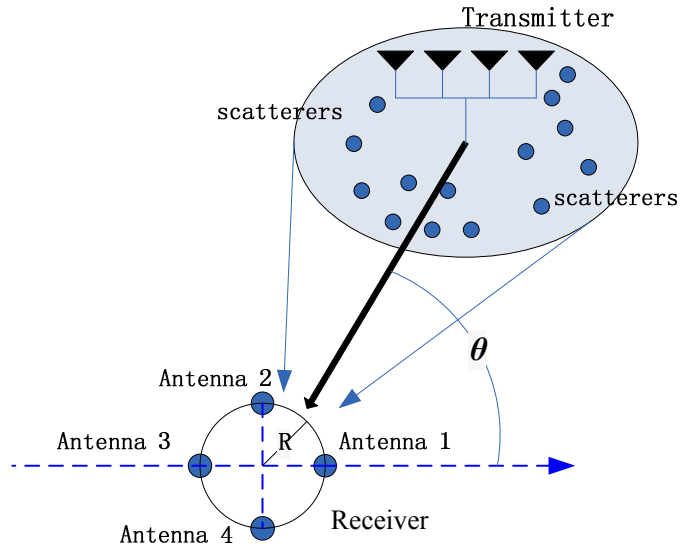

(a). UCA

$$
C_{l}=\frac{a}{2\left(1-e^{-a \pi}\right)}
$$

with $a$ representing a decay factor related to the angle spread (AS). When $a$ increases the angle spread decreases. $J_{n}($.$) is an n$-th order Bessel function of the first kind. $Z_{c}$ is related to the antenna spacing and $\alpha$ is the relative angle between the $m$-th and $n$-th antenna. If we let

$$
\begin{aligned}
& K 1=2 \pi \frac{R}{\lambda}\left[\cos \left(\varphi_{m}\right)-\cos \left(\varphi_{n}\right)\right] \\
& K 2=2 \pi \frac{R}{\lambda}\left[\sin \left(\varphi_{m}\right)-\sin \left(\varphi_{n}\right)\right]
\end{aligned}
$$

where $\varphi_{m}$ is the angle of $m$-th antenna in azimuthal planes, then:

$$
\begin{aligned}
& \sin (\alpha)=\frac{K 1}{\sqrt{K 1^{2}+K 2^{2}}} \\
& \cos (\alpha)=\frac{K 2}{\sqrt{K 1^{2}+K 2^{2}}} \\
& Z_{c}=\sqrt{K 1^{2}+K 2^{2}}
\end{aligned}
$$

The mathematical expressions for real and imaginary components of spatial correlation between $m$-th and $n$-th antenna at the receiver for the case of ULA antenna are given as [4]:

$$
\begin{aligned}
& \operatorname{Re}\{\operatorname{Rr}(m, n)\}=J_{0}\left(Z_{c}\right)+ \\
& 2 \sum_{k=1}^{\infty} \frac{a^{2}\left(1-e^{-a \pi}\right)}{a^{2}+4 k^{2}} J_{2 k}\left(Z_{c}\right) \cos (2 k \theta) \\
& \operatorname{Im}\{\operatorname{Rr}(m, n)\}=4 C_{l} \sum_{k=0}^{\infty} \frac{a^{2}\left(1+e^{-a \pi}\right)}{a^{2}+(2 k+1)^{2}} \\
& J_{2 k+1}\left(Z_{l}\right) \sin [(2 k+1) \theta]
\end{aligned}
$$

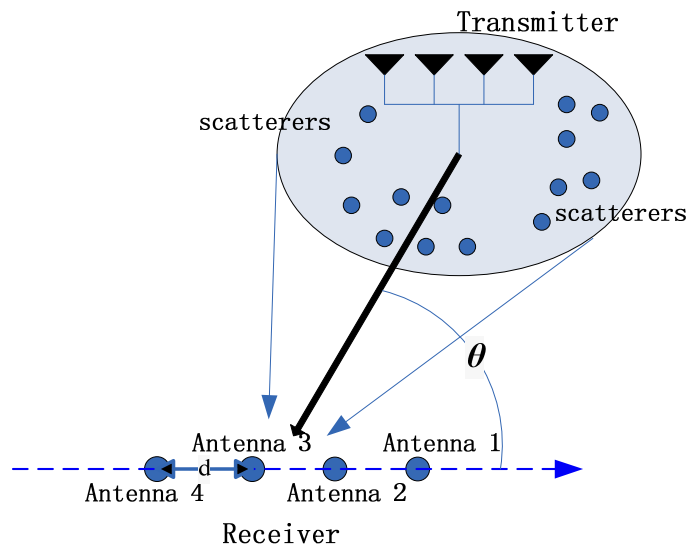

(b). ULA

Figure 1. 4-element UCA and ULA. 


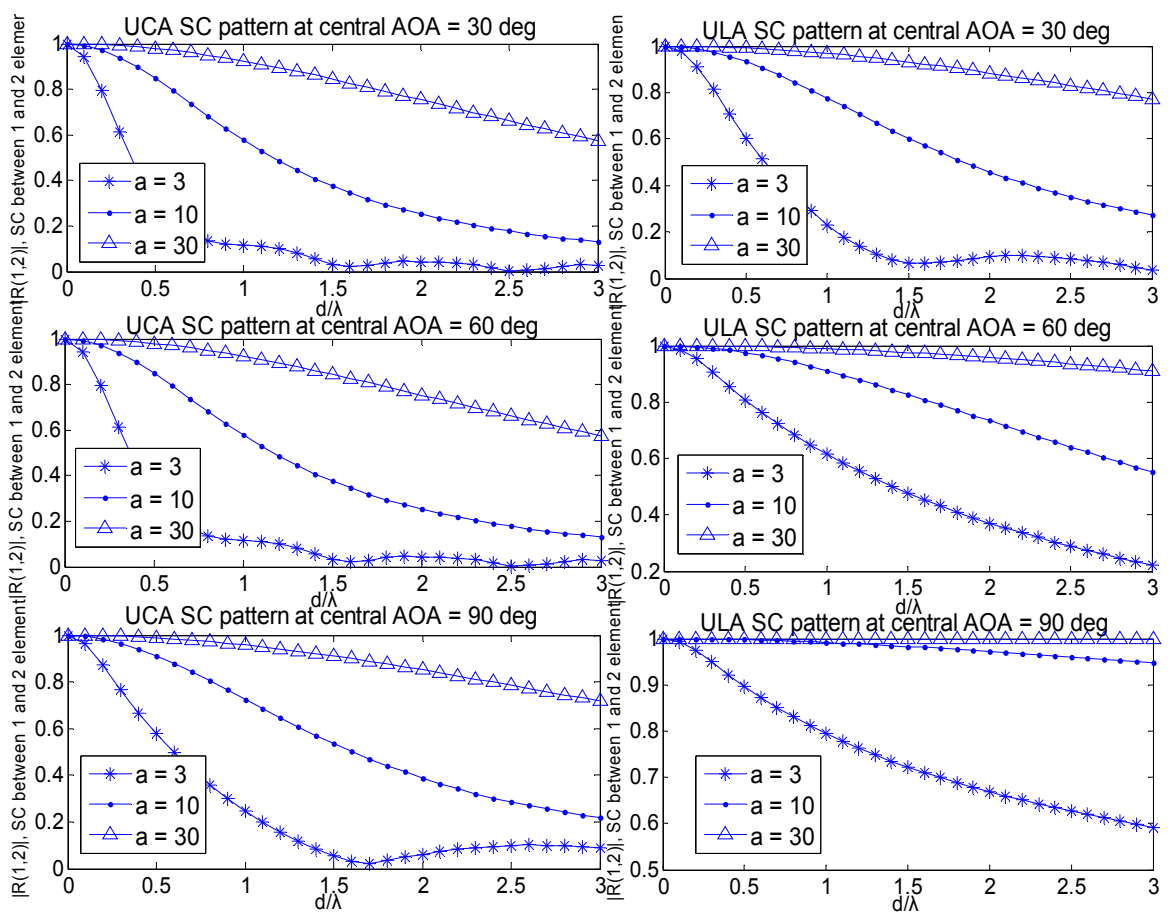

Figure 2. Spatial correlation between antenna 1 and 2 for UCA and ULA at AOA of $30^{\circ}, 60^{\circ}$ and $90^{\circ}$.

where $Z_{l}=2 \pi(m-n) d / \lambda$ and $d$ is antenna spacing.

The above expressions (1) (2) and (7) (8) can be applied to determine spatial correlations between any two antenna elements in UCA or ULA receiving antennas. Note that these expressions do not include the effect of antenna mutual coupling. This condition is approximately fulfilled when the antenna element spacing is about half of the wavelength or more.

Figure 2 shows the spatial correlation between two antenna elements (1 and 2) of a UCA or ULA antenna when the central AOA is $30^{\circ}, 60^{\circ}$ and $90^{\circ}$. There are three curves in each plot. These curves correspond to a different decay factor $a$ of 3,10 and 30 .

From the results presented in Figure 2, it is apparent that for the same antenna spacing $d / \lambda$ the spatial correlation in ULA is higher than that in UCA when the central AOA increases from $30^{\circ}$ to $90^{\circ}$. This can be due to the fact that ULA offers limited diversity when signals arrive from directions close to the ULA end-fire direction. UCA eliminates this deficiency as it offers almost a uniform view angle for all directions.

\subsection{Channel model}

A flat block-fading narrow-band MIMO system with $M_{t}$ array antennas at transmitter and $M_{r}$ array antennas at receiver is considered. The relationship between the received and transmitted signals is given by (9):

$$
Y_{s}=H s(t)+v(t)
$$

where $Y_{s}$ is the $M_{r} \times N$ complex matrix representing the received signals; $s(t)$ is the $M_{t} \times N$ complex matrix representing transmitted signals at time domain $t ; H$ is the $M_{r} \times M_{t}$ complex channel matrix and $v(t)$ is the $M_{r} \times N$ complex zero-mean white noise matrix at time domain $t$. $N$ is the length of transmitted signal. The channel matrix $H$ describes the channel properties which depend on antenna array configuration and signal propagation environment.

In order to simulate properties of the MIMO channel we apply the Kronecker model [5,6]. In this model, the transmitter and receiver correlations are assumed to be separable and the channel matrix $H$ is represented as:

$$
H=R_{R}^{1 / 2} H_{g} R_{T}^{1 / 2}
$$

where $H_{g}$ is a matrix with identical independent distributed (i.i.d) Gaussian entries with zero mean and unit variance and $R_{R}$ and $R_{T}$ are spatial correlation matrices at the receiver and transmitter, respectively. The channel correlation is expressed as,

$$
R_{H}=E\left\{H H^{H}\right\}
$$

where $\mathrm{E}\{\}$ stands for expected statistic value.

For the array configurations shown in Figure 1, the correlation experienced by pairs of transmitting antennas can be written as [7]:

$$
R_{t}(m, n)=J_{0}[2 \pi(m-n) / \lambda]
$$

Therefore, the correlation matrix $R_{t}$ for the MS trans- 
mitting antennas can be generated using (13)

$$
R_{t}=\left[\begin{array}{ccc}
R_{t}(1,1) & \cdots & R_{t}\left(1, M_{t}\right) \\
\vdots & \ddots & \vdots \\
R_{t}\left(M_{t}, 1\right) & \cdots & R_{t}\left(M_{t}, M_{t}\right)
\end{array}\right]
$$

In turn, the correlation matrix for the receiving antennas, $R_{r}$, can be obtained using Equations (1) (2) and (7) (8) and can be shown to be given as (14).

$$
R_{r}=\left[\begin{array}{ccc}
R_{r}(1,1) & \cdots & R_{r}\left(1, M_{r}\right) \\
\vdots & \ddots & \vdots \\
R_{r}\left(M_{r}, 1\right) & \cdots & R_{r}\left(M_{r}, M_{r}\right)
\end{array}\right]
$$

Having determined $R_{t}$ and $R_{r}$, the channel matrix $H$ can be calculated using Equation (10).

\section{BER Performance with BPSK Modulation Scheme}

\subsection{BER Performance Analysis}

Under a fading channel scenario, the average error probability can be obtained by averaging the conditional error probability over the probability density function (pdf) of instantaneous SNR $\gamma$ as:

$$
P_{e}=\int_{0}^{\infty} P(e \mid \gamma) P(\gamma) d \gamma
$$

To obtain $p(\gamma)$, the method in [14] can be used to find the characteristic function $\Phi_{\gamma}$ of $\gamma$. By applying the operation of an inverse Fourier transform (IFT) to the characteristic function, $p(\gamma)$ can be derived. According to [1], the general expression for the characteristic function of $\gamma$ is given as:

$$
\Phi_{\gamma}(w)=\left|I_{M_{r}}-n w \frac{\gamma}{m} R\right|^{-m}
$$

In which, $w=\mathrm{t} / \rho$ and $\rho$ is the transmit SNR; $m$ indicates the fading distribution properties. Such as $m=1$ and $m=1 / 2$ corresponds Rayleigh and the one-sided Gaussian distribution, respectively; $R$ is the channel correlation matrix.

Here, we assume the modulation schemes for the MIMO system under investigation are differential binary phase-shift key (DBPSK) and binary orthogonal frequency-shift key (BFSK). In the noncoherent case, the conditional BER for DBPSK and BFSK is given as [8],

$$
P(e \mid \gamma)=\frac{1}{2} \exp (-\alpha \gamma)
$$

in which $\alpha$ is modulation constant. BFSK corresponds to $\alpha=1 / 2$ and DBPSK corresponds to $\alpha=1$. The average BER can be written as:

$$
\begin{aligned}
P_{e}^{\text {noncoherent }} & =\frac{1}{2} \int_{0}^{\infty} \exp (-\alpha \gamma) P(\gamma) d \gamma \\
& =\left.\frac{1}{2} \Phi_{\gamma}(t)\right|_{n t=-\alpha} \\
& =\frac{1}{2}\left|I_{M_{r}}+\frac{\alpha \gamma}{m} R\right|^{-m}
\end{aligned}
$$

In turn, for the noncoherent case, the conditional BER for DBPSK and BFSK is given as [8],

$$
P(e \mid \gamma)=Q(\sqrt{2 \alpha \gamma})
$$

in which, $Q(\mathrm{x})$ is Gaussian $\mathrm{Q}$ function and is expressed as [9],

$$
Q(x)=\frac{1}{\pi} \int_{0}^{\pi / 2} \exp \left(-\frac{x^{2}}{2 \sin ^{2} \theta}\right) d \theta, \quad x \geq 0
$$

The average BER can be written as

$$
\begin{aligned}
P_{e}^{\text {coherent }} & =\frac{1}{2} \int_{0}^{\infty} \int_{0}^{\pi / 2} \exp \left(-\frac{\alpha \gamma}{\sin ^{2} \theta}\right) d \theta P(\gamma) d \gamma \\
= & \left.\frac{1}{\pi} \int_{0}^{\pi / 2} \Phi_{\gamma}(t)\right|_{n t=-\alpha / \sin ^{2} \theta} \\
= & \frac{1}{\pi}\left|I_{M_{r}}+\frac{\alpha \gamma}{m \sin ^{2} \theta} R\right|^{-m} d \theta
\end{aligned}
$$

\subsection{Numerical Results}

For the convenience of simulation, average BER of noncoherent DBPSK and BFSK are applied to evaluate the BER performance for the MIMO system. We assume that a ULA is present at the transmitter and either a UCA or ULA is located at the receiver. Simulations are performed for different values of the central AOA, decay factor, SNR and varying numbers of transmit/receive antennas.

In the first scenario, 4-element array antennas are used at both the transmitter and receiver of a MIMO system. The spacing $d$ between adjacent elements of ULA or the radius $R$ of UCA at transmitter is set at 0.5 wavelength $(\lambda)$. To reduce the antenna mutual coupling (which is neglected here) and correlation, $d$ and $R$ can be made larger than $0.5 \lambda$. Figures 3, 4, 5 and 6 show BER as a function of SNR for both UCA and ULA for three values of decay factor $a$, and for the central AOA equal to $0^{\circ}$, $30^{\circ}, 60^{\circ}$ and $90^{\circ}$. The modulation schemes are noncoherent BFSK and DBPSK.

The presented results indicate that BER decreases when SNR increases. At a higher decay factor, BER performances are worse. This can be explained by the fact that a larger decay factor corresponds to a smaller angle spread (AS) indicating a higher spatial correlation level. BER performances are degraded due to correlation.

In Figures 3 and 4, one can see that for the central AOA of $0^{\circ}$ and $30^{\circ}$ BER for both BFSK and DBPSK for ULA are better than for UCA for the three chosen values of decay factor; the BER performance of DBPSK is always 


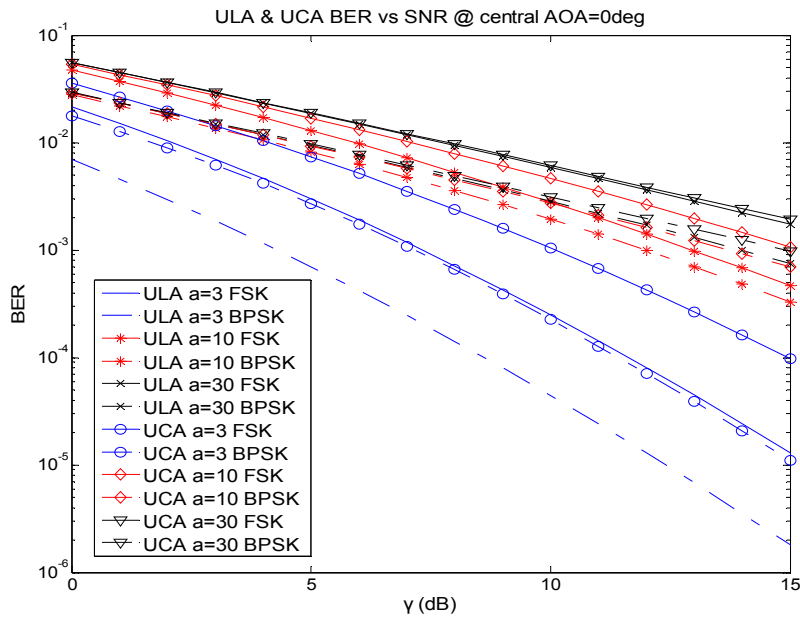

Figure 3. Noncoherent FSK and BPSK BER of UCA and ULA vs SNR at central $A O A=0^{\circ}$ for three values of decay factor $a$ of 3,10 and 30 .

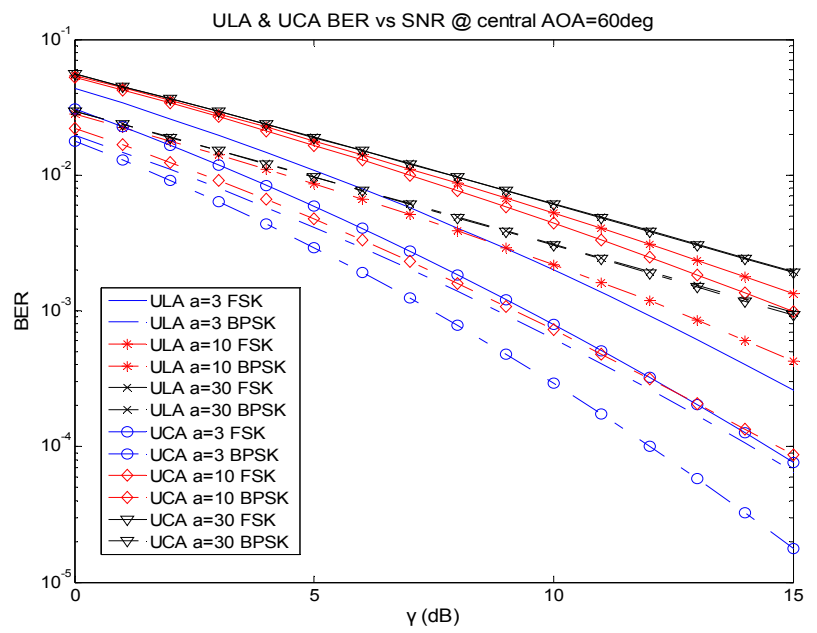

Figure 5. Noncoherent FSK and BPSK BER of UCA and ULA vs SNR at central $A O A=60^{\circ}$ for three values of decay factor $a$ of 3,10 and 30 .

better than BFSK.

However, when the central AOA is increased to $60^{\circ}$ and $90^{\circ}$ an opposite result is observed in Figures 5 and 6 . In the latter case, performance of UCA is superior in comparison with ULA. These opposite trends indicate that at a certain value of central AOA, the performances of UCA and ULA should be equal.

In order to determine the cross point (for BER) further simulations are performed. The results are shown in Figure 7. BER is presented in unit of $\mathrm{dB}$. One can see in Figure 7 that BER for ULA increases when the central AOA increases. This is because the ULA's spatial correlation level increases as the central AOA gets larger. While the BER curve for UCA is almost constant through the central AOA range. The cross point is be-

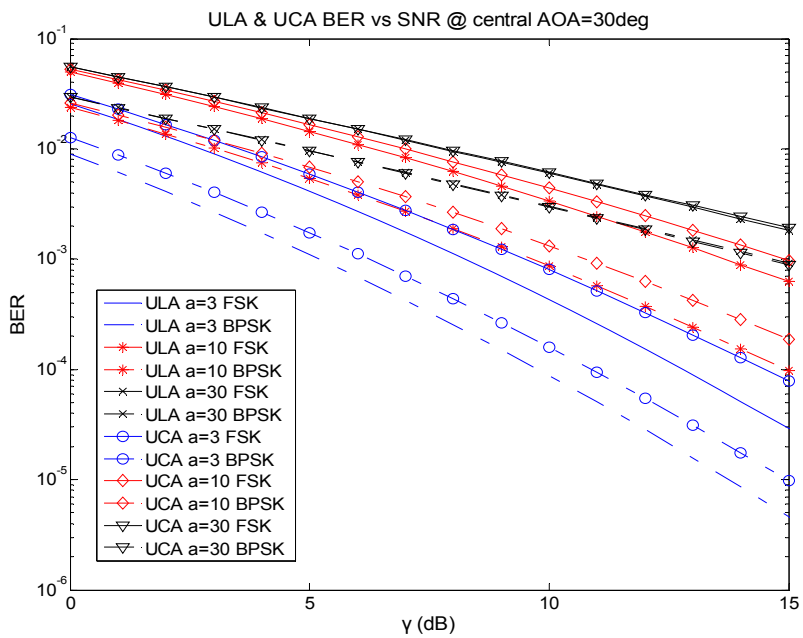

Figure 4. Noncoherent FSK and BPSK BER of UCA and ULA vs SNR at central $A O A=30^{\circ}$ for three values of decay factor $a$ of 3,10 and 30 .

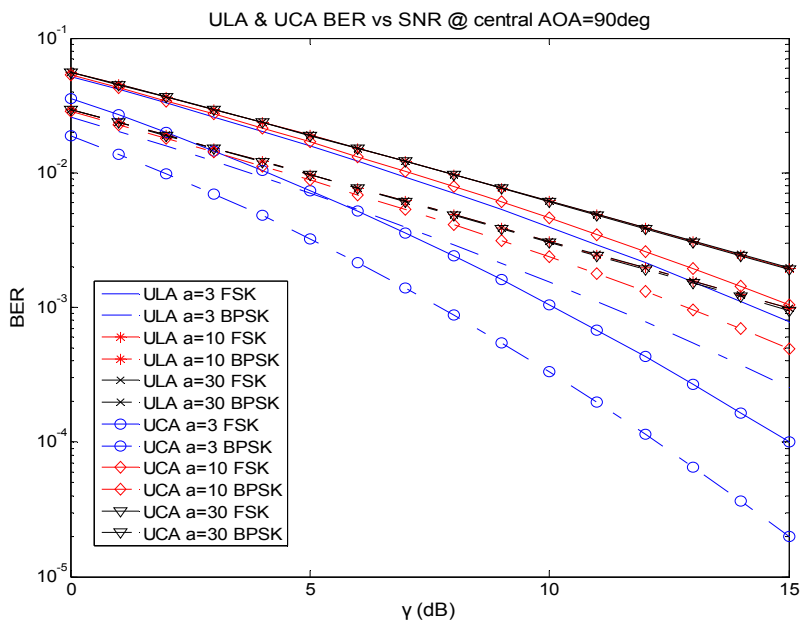

Figure 6. Noncoherent FSK and BPSK BER of UCA and ULA vs SNR at central $A O A=90^{\circ}$ for three values of decay factor $a$ of 3,10 and 30 .

tween approximate $\mathrm{AOA}=40^{\circ}$ and $\mathrm{AOA}=50^{\circ}$. To the left of the cross point, BER of ULA is lower than the one for UCA. In turn, on the right hand side, UCA's performance is better.

Using the earlier described settings for the $4 \times 4$ MIMO, the number of receiving antenna elements is increased and then the spatial correlation patterns and channel capacity versus the number of transmit and receive antennas are simulated.

Because of a usually small size of the mobile station, the number of transmitting antennas is limited. This is not the case of base station which offers a larger available area where more antennas can be added. Here, the number of antenna elements in a ULA is assumed to increase along the line with same spacing $d$, as shown in 


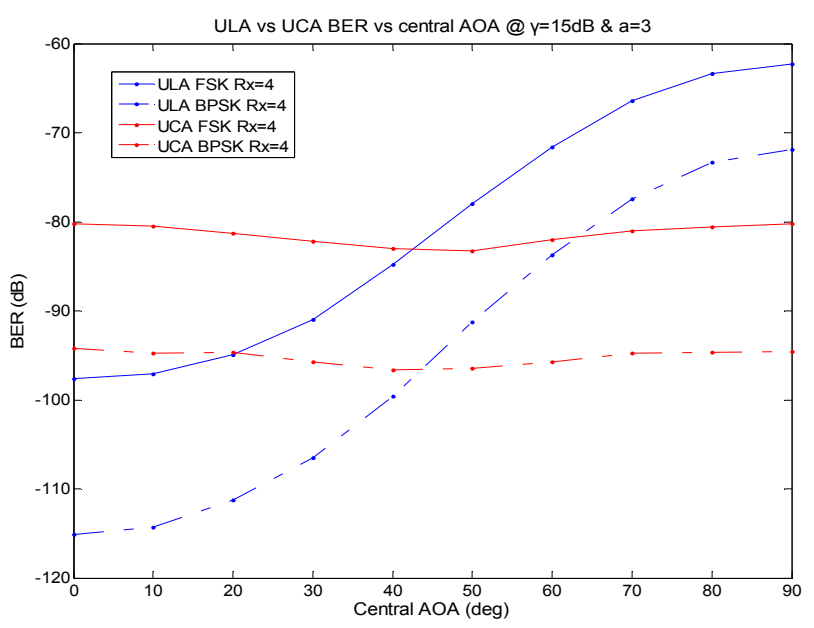

Figure 7. Noncoherent BFSK \& DBPSK BER of UCA and ULA vs central AOA.

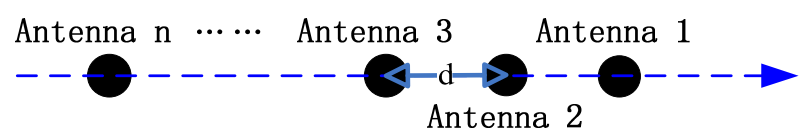

(a). ULA

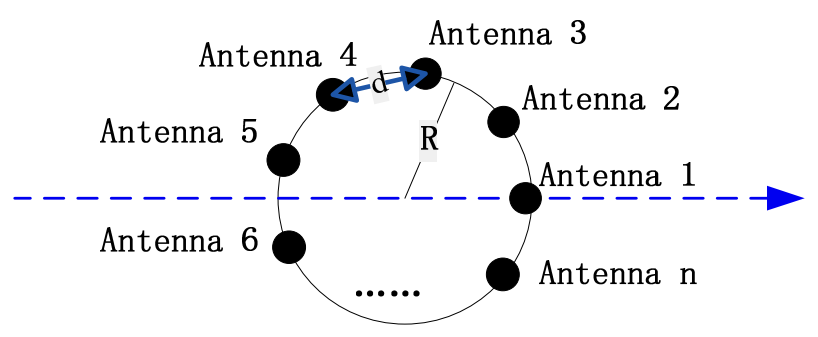

(b). UCA

Figure 8. ULA and UCA antenna arrays.

Figure 8(a). In turn, for a UCA the number of antenna elements with the same spacing $d$ increases on the circle, as shown in Figure 8(b). In the UCA case, when the number of elements on the circle increases with spacing $\mathrm{d}$ unchanged, the radius $\mathrm{R}$ increases correspondingly.

Figure 9 presents the spatial correlation between antenna elements 1 and 2 for receiving UCA and ULA for the new settings. From Figure 9(a), one can see that when the number of antenna elements increases the spatial correlation for UCA varies from 0 to 1 . However, the variation due to an increased number of antenna elements is very small. For the ULA case, the spatial correlation level of receiving antennas is unchanged when the number of antennas increases.

Similarly as Figures 3-6, Figures 10, 11 and 12 show the results of BER for a different number of receiving antenna elements for the cases of ULA and UCA receiveing
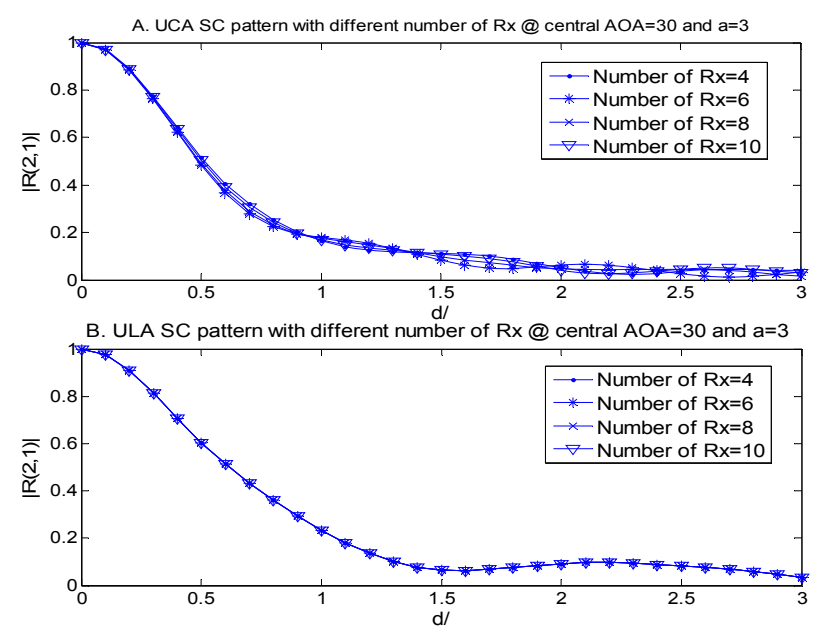

Figure 9. Spatial correlation between antenna 1 and antenna for different numbers $(4,6,8$,and 10$)$ of antenna elements in receiving UCA (A) and ULA (B) antenna arrays at central $\mathrm{AOA}$ of $30^{\circ}$ and decay factor $a$ of 3 .

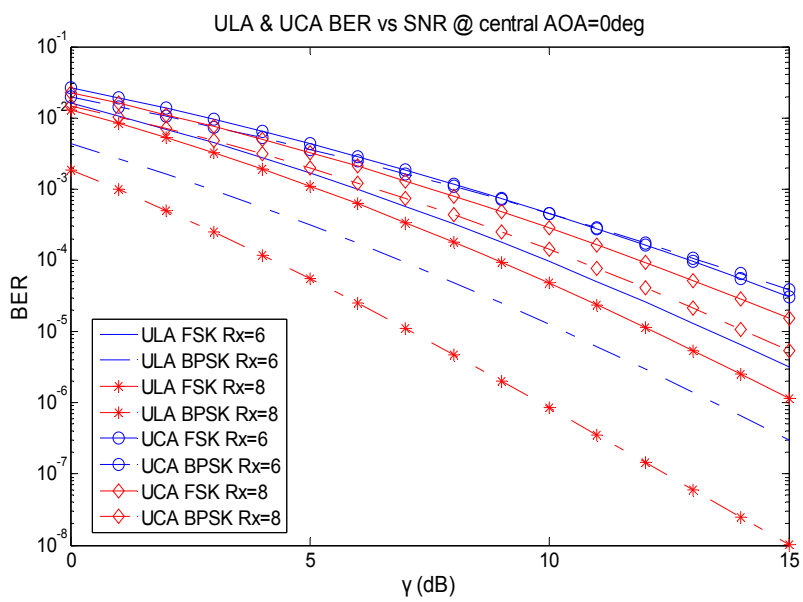

Figure 10. ULA vs UCA BER with different number of Rx antenna array elements at decay factor $a$ equal to 3 and central AOA equal to $0^{\circ}$.

antennas. An increase in the number of antenna elements in ULA and UCA brings improvement to the BER performance. The cross points between red curves representing ULA and blue curves standing for UCA move to the right from approximate $40^{\circ}$ to $50^{\circ}$ as the number of antennas increases.

\section{MIMO Channel Capacity with Perfect Knowledge of Channel Matrix}

\subsection{MIMO Channel Capacity \& EDOF}

If CSI is perfectly known at the receiver but unknown at the transmitter, the capacity of a MIMO system with $M_{r}$ receive antennas and $M_{t}$ transmit antennas can be deter- 
mined using $[10,11]$ :

$$
C=E\left(\log _{2}\left\{\operatorname{det}\left[I_{M_{R}}+\frac{\rho}{M_{t}}\left(H H^{H}\right)\right]\right\}\right)
$$

where $\{.\}^{\mathrm{H}}$ stands for the transpose-conjugate; $\rho$ is the total transmitted SNR.

An alternative expression for the capacity in such a case can be obtained by decomposing the channel into $n$ $=\min \left(M_{r}, M_{t}\right)$ virtual single input single output (SISO) sub-channels, and can be shown to be given as (23),

$$
C=\sum_{i}^{n} \log _{2}\left(1+\frac{\rho_{i}}{n} \lambda_{i}\right)
$$

where

$$
\rho=\sum_{i}^{n} \rho_{i}
$$

and the gains of sub-channels are represented by the eigenvalues of the channel correlation matrix $\mathrm{HH}^{H}$.

Here, it is assumed that the transmitted power is equally allocated to each sub-channel, which is easy to accomplish in practice. The channel capacity can be further maximized by applying power allocation schemes such as 'water-filling'. However, this is not easy to implement, as CSI is required at the transmitter, which must be sent from the receiver to the transmitter.

It has to be noted that the MIMO channel capacity can be related to the channel effective degree of freedom (EDOF) [12]. In order to determine EDOF, the channel matrix properties and the signal to noise ratio (SNR) are required. According to [12], the EDOF is defined as:

$$
\left.E D O F \equiv \frac{d}{d \delta} C\left(2^{\delta} \rho\right)\right|_{\delta=0}
$$

Given the eigenvalues of the channel correlation $H H^{H}$, it can be rewritten as

$$
\left.\frac{d}{d \delta} C\left(2^{\delta} \rho\right)\right|_{\delta=0}=\left.\frac{d}{d \delta} \sum_{i}^{n}\left[\log _{2}\left(1+\frac{2^{\delta} \rho}{n} \lambda_{i}\right)\right]\right|_{\delta=0}=\sum_{i}^{n} \frac{\frac{\rho}{n} \lambda_{i}}{1+\frac{\rho}{n} \lambda_{i}}
$$

It is apparent that when $\rho \lambda_{i} / n>>1,(18)$ is approximately equal to $n$ and EDOF becomes maximum. In this case, every sub-channel is useful to transmit signals. In turn, when $\rho \lambda_{i} / n<1$, EDOF is smaller than $n$, some sub-channels are not efficient to transmit signals. Rea sons for the reduced EDOF can be due to an increased level of channel correlation and decreased SNR.

\subsection{Numerical Results}

Based on the presented theory, the channel EDOF and capacity are simulated for a $4 \times 4$ MIMO system. The spacing $d$ between adjacent elements of ULA or the radius $R$ of UCA at transmitter is set at 0.5 wavelength $(\lambda)$. Figures 13, 14, 15 and 16 show EDOF and capacity as a function of SNR for both UCA and ULA for three values of decay factor $a$, and for the central AOA equal to $0^{\circ}$, $30^{\circ}, 60^{\circ}$ and $90^{\circ}$.

The presented results reveal that both EDOF and capacity increase when SNR increases. At a higher decay factor, both EDOF and capacity are lower. This is because of the fact that a larger decay factor corresponds to a smaller angle spread (AS) indicating a higher spatial correlation level. EDOF and capacity are degraded due to correlation.

In Figures 13 and 14, one can see that for the central AOA of $0^{\circ}$ and $30^{\circ}$ both EDOF and capacity for ULA are higher than for UCA for the three chosen values of decay factor.

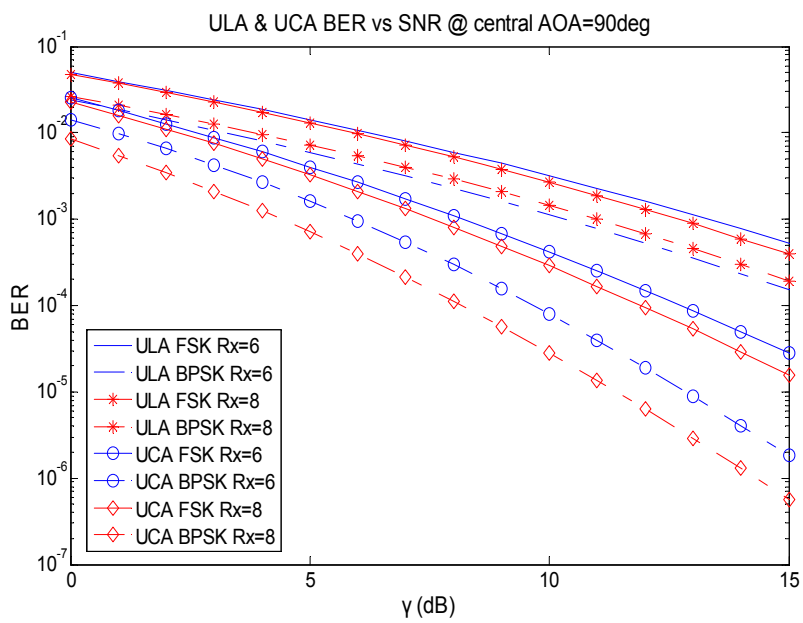

Figure 11. ULA vs UCA BER with different number of Rx antenna array elements at decay factor $a$ equal to 3 and central AOA equal to $90^{\circ}$.

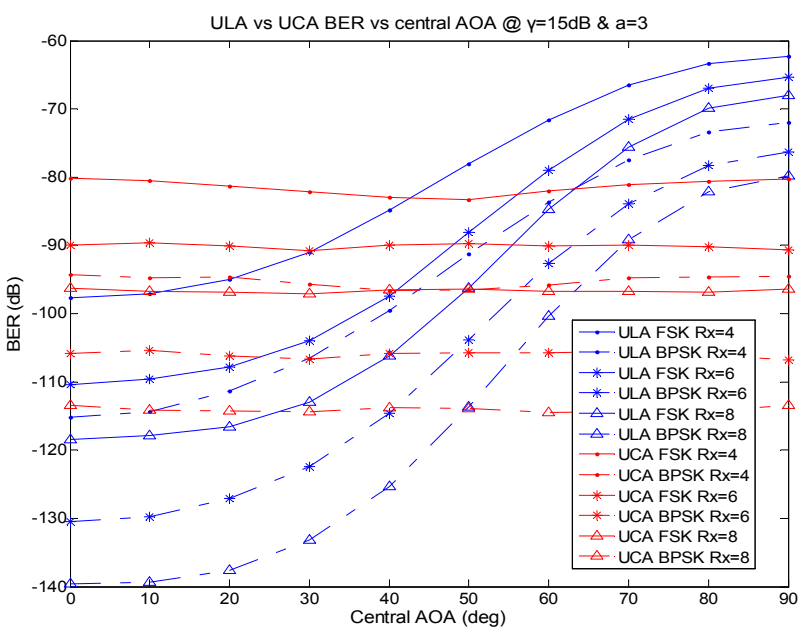

Figure 12. ULA vs UCA BER with different number of Rx antenna array elements decay factor $a$ equal to 3 . 

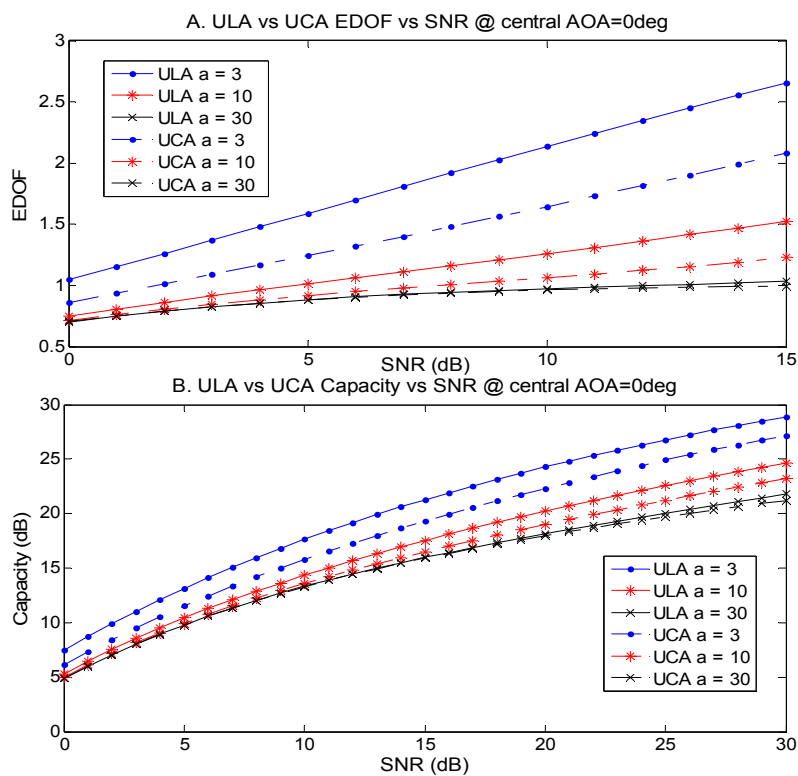

Figure 13. EDOF and capacity of UCA and ULA vs SNR at central $\mathrm{AOA}=0^{\circ}$ for three values of decay factor $a$ of 3,10 and 30.
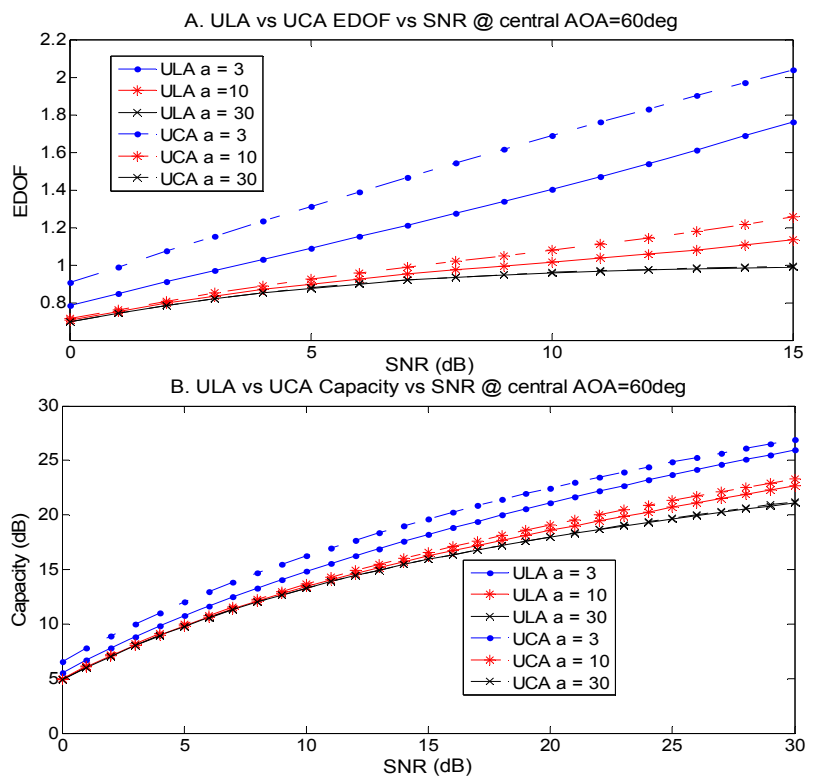

Figure 15. EDOF and capacity of UCA and ULA vs SNR at central $\mathrm{AOA}=60^{\circ}$ for three values of decay factor $a$ of 3,10 and 30.

When the central AOA is increased to $60^{\circ}$ and $90^{\circ}$ an opposite result is observed in Figure 5 and 6. In the latter case, performance of UCA is superior in comparison with ULA.

To determine the cross point (for EDOF or capacity) further simulations are performed. The results are shown in Figure 17. One can see in Figure 17 that both EDOF and capacity decrease for the case of ULA when the central AOA increases at two different SNR. This is because the ULA's spatial correlation level increases as the central
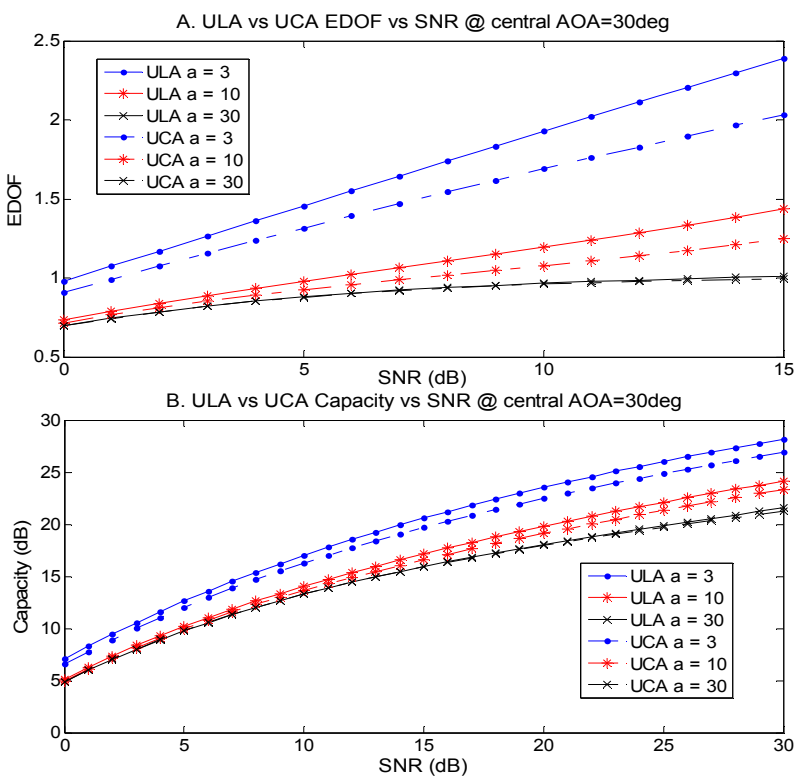

Figure 14. EDOF and capacity of UCA and ULA vs SNR at central $\mathrm{AOA}=30^{\circ}$ for three values of decay factor $a$ of 3,10 and 30.
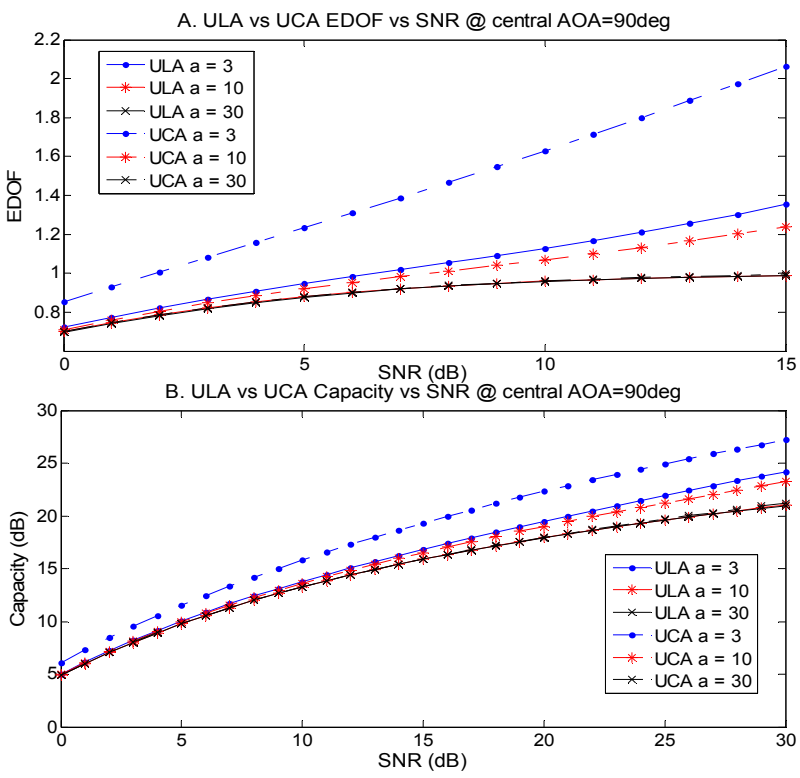

Figure 16. EDOF and capacity of UCA and ULA vs SNR at central $\mathrm{AOA}=90^{\circ}$ for three values of decay factor $a$ of 3,10 and 30.

AOA gets larger. This degrades channel capacity. The cross point is between $\mathrm{AOA}=40^{\circ}$ and $\mathrm{AOA}=50^{\circ}$. To the left of the cross point, EDOF and capacity of ULA is higher than for UCA. In turn, on the right hand side, UCA's performance is better.

Figures 18, 19 and 20 show the results for channel capacity for a different number of receiving antenna elements for the cases of ULA and UCA receiving antennas. An increase in the number of antenna elements in ULA and UCA brings improvement to the channel capacity. 
The cross points between red curves representing ULA and blue curves standing for UCA move to the right as the number of antennas increases. When the number of receiving antenna elements is 10 , the capacity of ULA is superior to UCA for the central AOA of $0^{\circ}$ to $50^{\circ}$.

\section{MIMO Channel Estimation}

For the training based channel estimation method, the relationship between the received signals and the training sequences is given by Equation (26) as

$$
Y=H P+V
$$
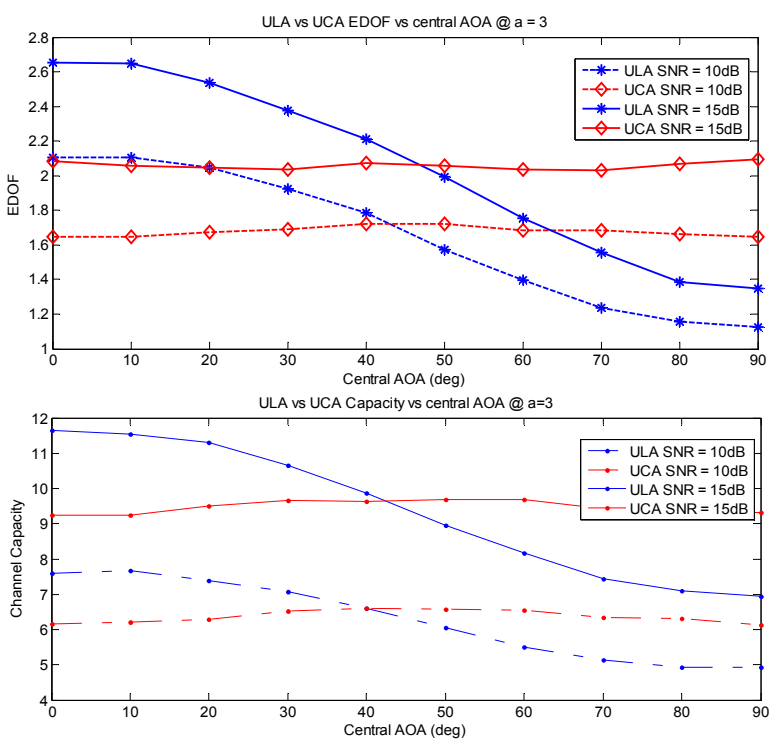

Figure 17. EDOF and capacity of UCA and ULA vs central AOA.

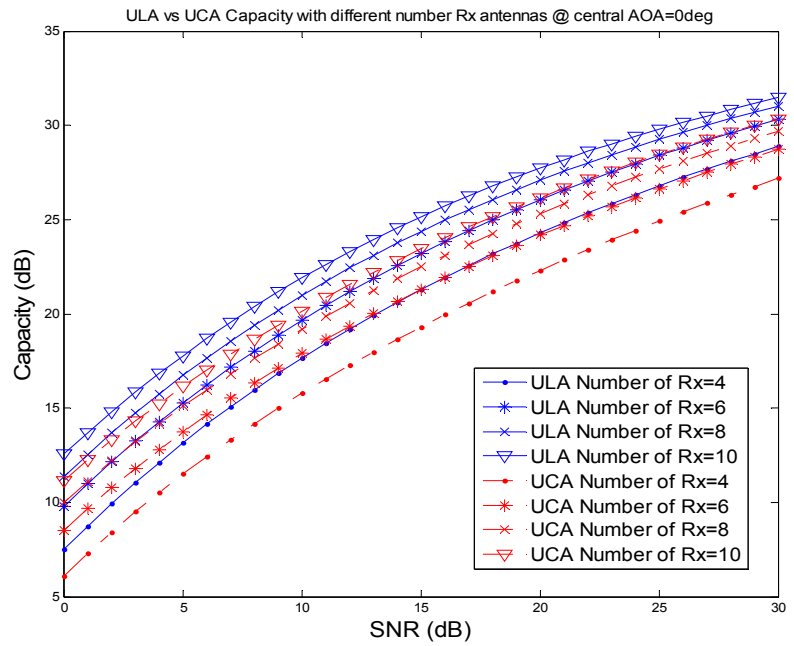

Figure 18. ULA vs UCA capacity with different number of Rx antenna array elements at decay factor $a$ equal to 3 and central AOA equal to $0^{\circ}$.

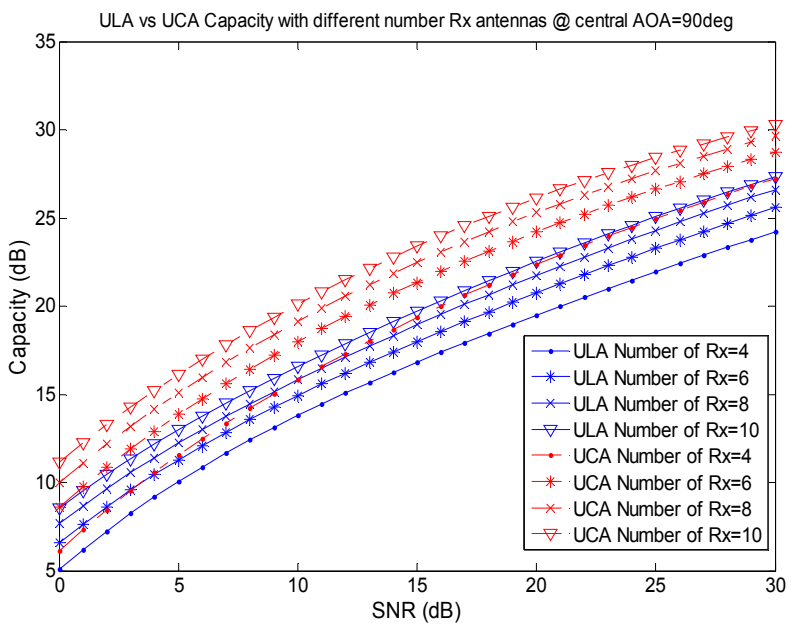

Figure 19. ULA (blue lines) vs UCA (red lines) capacity for different number of $\mathrm{Rx}$ antenna array elements at decay factor $a$ equal to 3 and central AOA equal to $90^{\circ}$.

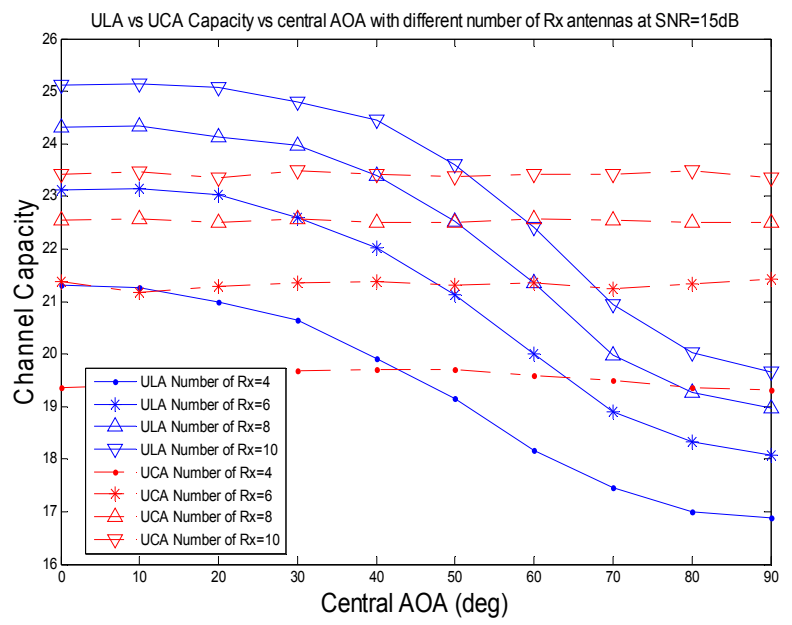

Figure 20. ULA vs UCA capacity with different number of $\mathbf{R x}$ antenna array elements decay factor $a$ equal to 3 .

Here the transmitted signal $S$ in (1) is replaced by $P$, which represents the $M_{t} \times L$ complex training matrix (sequence) where $L$ is the length of the training sequence. The goal is to estimate the complex channel matrix $H$ from the knowledge of $Y$ and $P$. The transmitted power in the training mode is assumed to be given by a constant value. According to [13] and [14], the estimation using SLS or MMSE method requires orthogonality of the training matrix $P$. In the undertaken analysis, the training matrix $P$ is assumed to satisfy this condition.

The performance of SLS method can be obtained by scaling up the results from the least square (LS) method. Using the LS method, the estimated channel can be written as $[13,14]$,

$$
\hat{H}_{L S}=Y P^{\dagger}
$$


where $\{.\}^{\dagger}$ stands for pseudo-inverse. The mean square error (MSE) of the LS method is given as

$$
M S E_{L S}=E\left\{\left\|H-\hat{H}_{L S}\right\|_{F}^{2}\right\}
$$

in which $E\{$.$\} denotes a statistical expectation. Accord-$ ing to [13] and [14], the minimum value of MSE for the LS method is given as

$$
M S E_{\min }^{L S}=\frac{M_{t}^{2} M_{r}}{\rho_{t}}
$$

in which $\rho^{t}$ stands for transmitted SNR in training mode.

The SLS method reduces the estimation error of the LS method and the improvement is given by the scaling factor $\gamma$ as

$$
\gamma=\frac{\operatorname{tr}\left\{R_{H}\right\}}{M S E_{L S}+\operatorname{tr}\left\{R_{H}\right\}}
$$

The estimated channel matrix is represented by $[13,14]$

$$
\hat{H}_{S L S}=\frac{\operatorname{tr}\left\{R_{H}\right\}}{\sigma_{n}^{2} M_{r}+\operatorname{tr}\left\{\left(P P^{H}\right)^{-1}\right\}+\operatorname{tr}\left\{R_{H}\right\}} Y P^{\dagger}
$$

Here, $\sigma_{n}{ }^{2}$ is the noise power; $R_{H}$ is the channel correlation matrix defined as $R_{H}=E\left\{H^{H} H\right\}$ and $\operatorname{tr}\{$.$\} implies the$ trace operation.

The MSE for SLS is given as $[13,14]$

$$
\begin{aligned}
M S E_{S L S} & =E\left\{\left\|H-\gamma \hat{H}_{L S}\right\|_{F}^{2}\right\} \\
& =(1-\gamma)^{2} \operatorname{tr}\left\{R_{H}\right\}+\gamma^{2} M S E_{L S}
\end{aligned}
$$

The minimized MSE of MMSE method can be written as $[7,8]$

$$
M S E_{\min }^{S L S}=\frac{M S E_{L S} \operatorname{tr}\left\{R_{H}\right\}}{M S E_{L S}+\operatorname{tr}\left\{R_{H}\right\}}
$$

By taking into account expression (23), the minimized MSE of the SLS method (27) can be rewritten as

$$
\begin{aligned}
M S E_{S L S} & =\left[\left(\operatorname{tr}\left\{R_{H}\right\}\right)^{-1}+\frac{\rho_{t}}{M_{t}^{2} M_{r}}\right]^{-1} \\
& =\left[\left(\sum_{i}^{n} \lambda_{i}\right)^{-1}+\frac{\rho_{t}}{M_{t}^{2} M_{r}}\right]^{-1}
\end{aligned}
$$

where $n=\min \left(M_{r}, M_{t}\right)$ and is $\lambda_{i}$ the $i$-th eigenvalue of the channel correlation $R_{H}$.

In the MMSE method, the estimated channel matrix is given as $(35)[13,14]$,

$$
\hat{H}_{M M S E}=Y\left(P^{H} R_{H} P+\sigma_{n}^{2} M_{r} I\right)^{-1} P^{H} R_{H}
$$

The MSE of MMSE estimation is given as

$$
M S E_{\text {MMSE }}=E\left\{\left\|H-\hat{H}_{M M S E}\right\|_{F}^{2}\right\}=\operatorname{tr}\left\{R_{E}\right\}
$$

in which $R_{E}$ is estimation error correlation written as

$$
\begin{aligned}
& R_{E}=E\left\{\left(H-\hat{H}_{M M S E}\right)\left(H-\hat{H}_{M M S E}\right)^{H}\right\} \\
& =\left(R_{H}^{-1}+\sigma_{n}^{-2} M_{r}^{-1} P P^{H}\right)^{-1}
\end{aligned}
$$

The minimized MSE for MMSE is obtained as [7,8]

$$
M S E_{\text {MMSE }}=\operatorname{tr}\left\{\left(\Lambda^{-1}+\sigma_{n}^{-2} M_{r}^{-1} Q^{H} P P^{H} Q\right)^{-1}\right\}
$$

In (38), $Q$ is the unitary eigenvector matrix of $R_{H}$ and $\Lambda$ is the diagonal matrix with eigenvalues of $R_{H}$. The minimized MSE for the MMSE method, given by Equation (38), can be rewritten using the orthogonality properties of the training sequence $P$ and the unitary matrix $Q$, as shown by

$$
\begin{aligned}
& M_{S} E_{\text {MMSE }}=\operatorname{tr}\left\{\left(\Lambda^{-1}+\rho_{t} M_{r}^{-1} I\right)^{-1}\right\} \\
& =\sum_{i}^{n}\left(\lambda_{i}^{-1}+\rho_{t} M_{r}^{-1}\right)^{-1}
\end{aligned}
$$

From Equations (33), (38) and (39), one can see that MSE of SLS and MMSE methods depends on the channel correlation which, in turn, is affected by the transmitter and receiver spatial correlations.

In the first instance, the SLS and MMSE channel estimation methods are assessed via computer simulations. In the undertaken simulations, the transmitter of the MIMO system is assumed to be equipped with ULA while the receiver uses either UCA or ULA. The case of 4x4 MIMO system is considered. The simulations are performed for different values of central AOA, decay factor $a$ and the transmitted SNR $\left(\rho_{t}=\rho\right)$. The other assumptions are similar to the ones already described in Subsection 3.2.

Simulations of MSE as a function of $\rho\left(\rho=P_{s} / \sigma_{n}{ }^{2}\right)$ for the SLS and MMSE channel estimation are performed for two decay factors of 3 and 30 assuming the central AOA of $0^{\circ}, 30^{\circ}, 60^{\circ}$ and $90^{\circ}$. The results are shown in Figures 21, 22, 23 and 24.

In all of the cases presented in Figures 21, 22, 23 and 24 it is apparent that when $\rho$ increases MSE decreases for both SLS and MMSE irrespectively from the choice of decay factor. MSE of SLS looks to be independent of the decay factor. Also only negligible changes in MSE of SLS are observed when CLA replaces ULA at the receiver. However, MSE of MMSE is sensitive to the choice of decay factor and is smaller for larger decay factors.

With reference to the choice of the central AOA of $0^{\circ}$ and $30^{\circ}$ in Figures 21 and 22, one can see that MSE of MMSE for ULA is larger than for UCA.

This happens irrespectively of the choice of the decay factor value. However, in the case of central AOA of $60^{\circ}$ and $90^{\circ}$, shown in Figures 23 and 24, one can see that the opposite conclusion takes place. The MSE of MMSE for the UCA is getting greater than when the ULA is used at the receiver. 


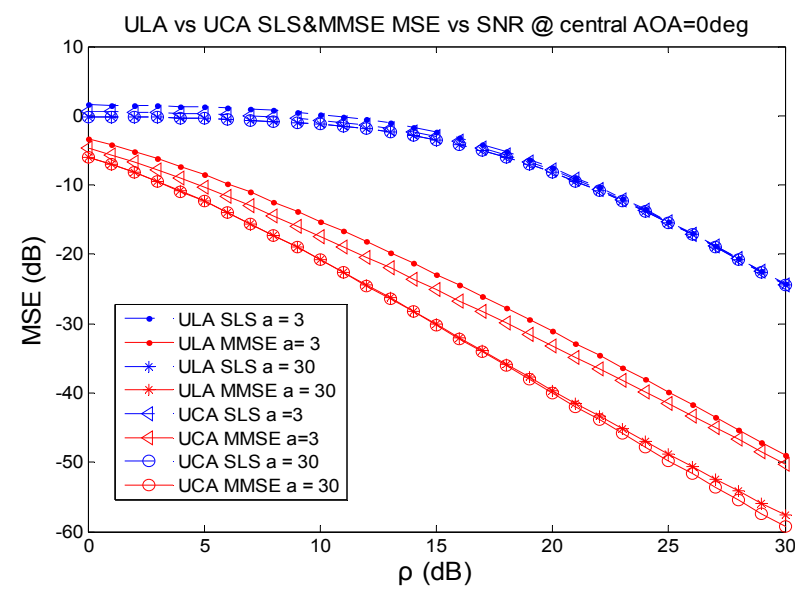

Figure 21. MSE vs $\rho$ for receiving ULA (blue lines) and UCA (red lines) at central $\mathrm{AOA}=0^{\circ}$.

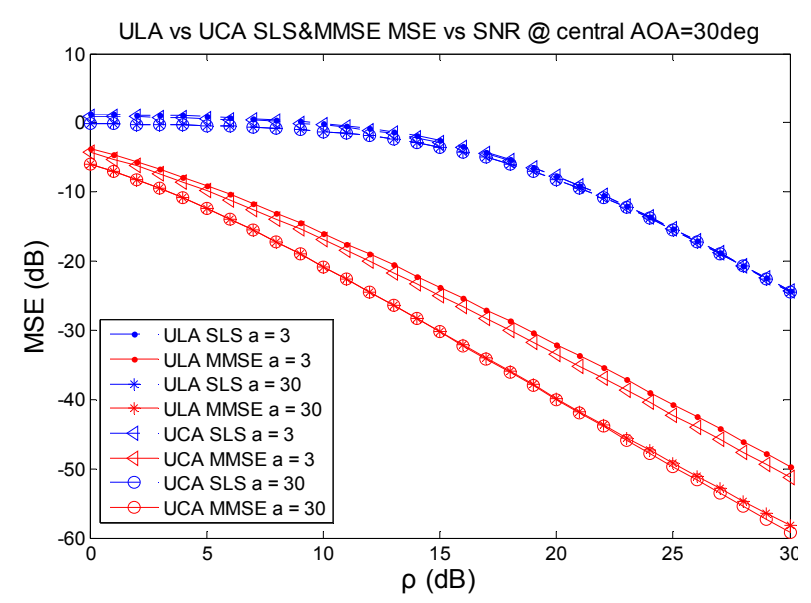

Figure 22. MSE vs $\rho$ for UCA and ULA at central AOA $=30^{\circ}$.

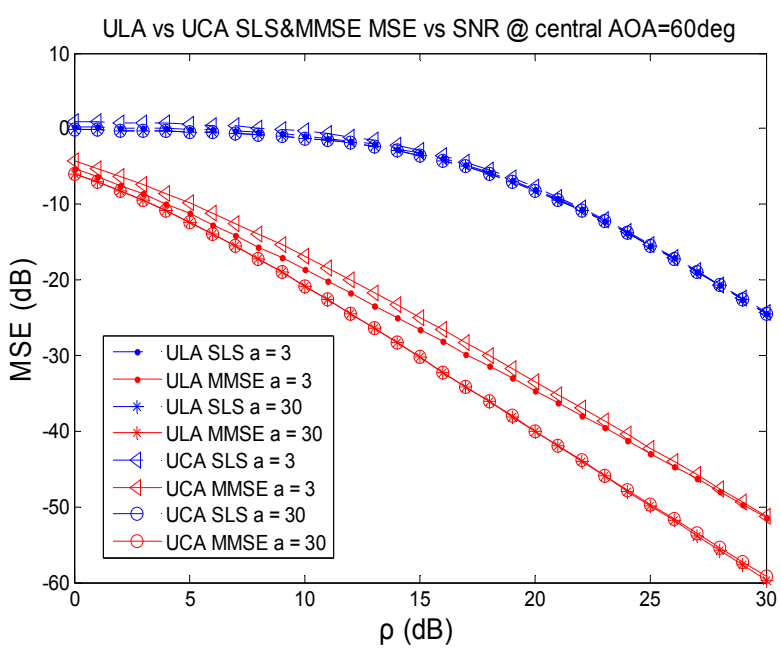

Figure 23. MSE vs $\rho$ for receiving ULA (blue lines) and UCA (red lines) at central $\mathrm{AOA}=60^{\circ}$.

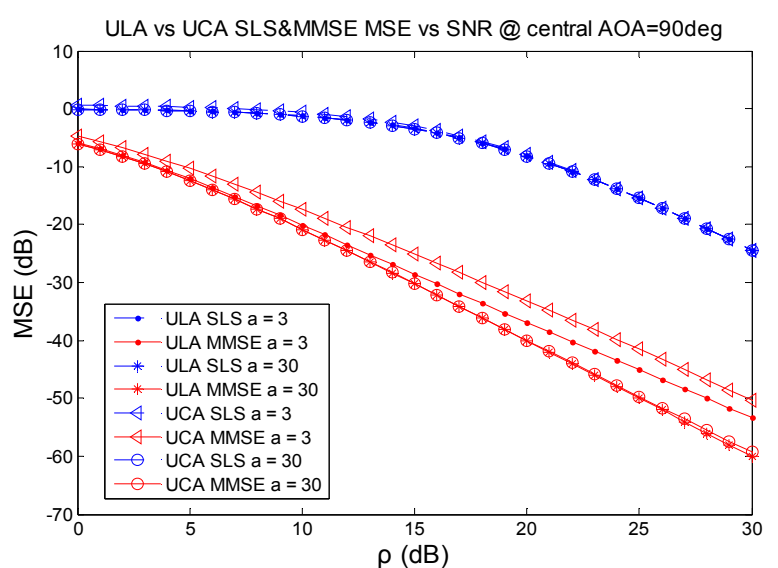

Figure 24. MSE vs $\rho$ for receiving ULA (blue lines) and UCA (red lines) at central $\mathrm{AOA}=\mathbf{9 0}^{\circ}$.

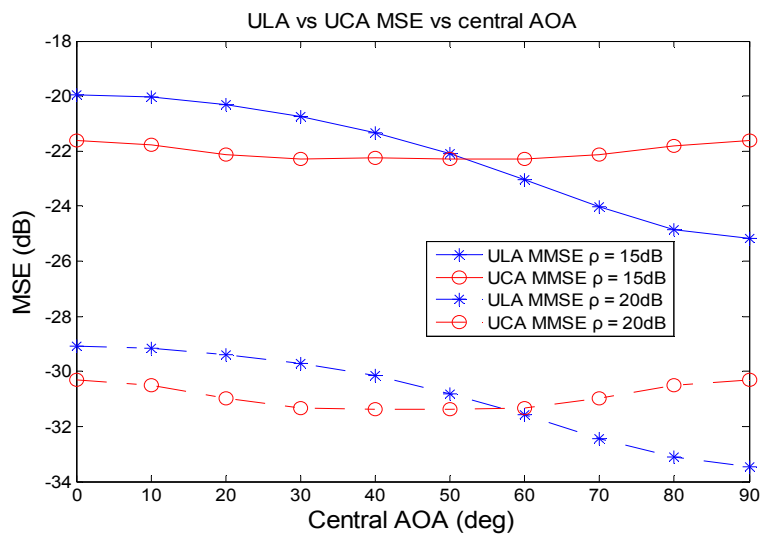

Figure 25. MSE vs central AOA for ULA (blue line) and UCA (red line) at decay factor $a$ equal to 3 .

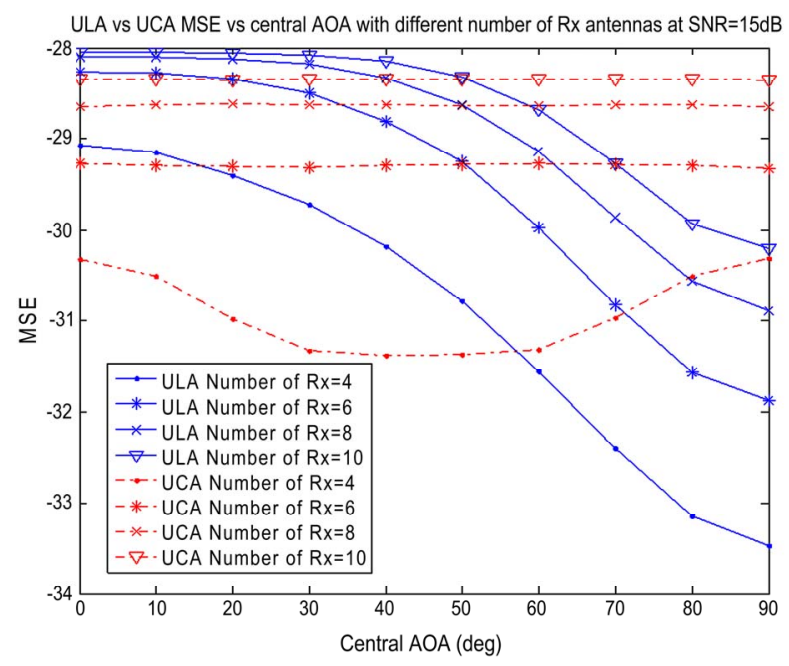

Figure 26. MSE vs central AOA for different number of antenna elements in receiving ULA and UCA at decay factor $a$ equal to 3 and $\rho$ equal to $20 \mathrm{~dB}$. 
Figure 25 shows the simulated results for MSE versus central AOA for two cases of $\rho$ equal to $15 \mathrm{~dB}$ and $20 \mathrm{~dB}$, respectively. One can see that when $\rho$ is equal to $15 \mathrm{~dB}$, MSE of MMSE for ULA is larger than for UCA when the central AOA is smaller than $50^{\circ}$. In turn, when the central AOA is larger than $50^{\circ}$ an opposite situation takes place: MSE of MMSE for UCA is larger than the one for ULA.

Similar observations are made when $\rho$ is equal to $20 \mathrm{~dB}$. However, in this case the central AOA cross point is moved to about $60^{\circ}$.

Figure 26 shows the results for MSE similar to those of Figure 25. However, they are obtained for different number of receiving antenna elements of 4, 6, 8 and 10 .

It can be seen in Figure 26 that when the receiving array includes 4 antenna elements, the channel estimation shows the best performance for both ULA and UCA. When the number of antenna elements is increased from 4 to 6,8 and 10 , the channel estimation accuracy is getting worse for both ULA and UCA cases. These results confirm our expectation that larger size MIMO systems face the problem of decreased estimation of MIMO channel.

\section{Conclusions}

In this paper, we have reported on investigations into the performance of BER, channel capacity and channel estimation of a MIMO system employing Uniform Linear Array at the transmitter and either a Uniform Circular Array or Uniform Linear Array at the receiver. In the presented investigations, the transmitter is assumed to be surrounded by scattering objects while the receiver is postulated to be free of scatterers. The signal angle of arrival (AOA) has been assumed to follow the Laplacian distribution. The angle spread (AS) is characterized by the decay factor.

The attention has been paid to the effect of different spatial correlation in receiving linear and circular arrays. The obtained results have shown that for the central AOA varying from $0^{\circ}$ to $90^{\circ}$, UCA's spatial correlation pattern (as a function of element antenna spacing) is relatively constant while ULA's spatial correlation level increases; both UCA's and ULA's spatial correlation patterns are not sensitive to the increased number of array elements.

At a larger decay factor corresponding to a smaller angular spread (and thus a higher level of spatial correlation), the BER of both FSK and BPSK are increased for both the UCA and ULA receiving antenna cases. Simulation results also presented the variation of BER as a function of central AOA varying from $0^{\circ}$ to $90^{\circ}$ when the signal to noise ratio $\gamma$ is equal to $15 \mathrm{~dB}$. It has been shown that at $\gamma=15 \mathrm{~dB}$, BER for ULA is lower in comparison with UCA when the central AOA is smaller than $45^{\circ}$.
When central AOA becomes larger than $50^{\circ}$, the UCA performance is better in terms of lower value of BER. When the number of receiving antennas increases, the performance gets better in terms of BER for both ULA and UCA cases.

The obtained results have also shown that for a larger decay factor, the channel capacity is reduced for both UCA and ULA receiving antennas. The $4 \times 4$ MIMO system employing the receiving ULA shows higher capacity when the central AOA is smaller than $40^{\circ}$. For central $\mathrm{AOA}$ greater than $50^{\circ}$ the opposite happens and the system using UCA outperforms the one using ULA. When the number of receiving antennas increases, improvements to channel capacity are demonstrated for both ULA and UCA. The cross points for ULA and UCA capacity curves move to the right when the number of antennas increases. When the number of receiving antennas is 10 , the capacity performance for ULA is superior to UCA for central AOA of $0^{\circ}$ to $50^{\circ}$.

For channel estimation performance, at a larger decay factor, the MSE of training based channel estimation methods such as SLS and MMSE is reduced for both the UCA and ULA receiving antenna cases. This agrees with the findings of [15] and [16]. Other presented results have concerned the variation of MSE as a function of central AOA varying from $0^{\circ}$ to $90^{\circ}$ when the signal to noise ratio $\rho$ is equal to $15 \mathrm{~dB}$ or $20 \mathrm{~dB}$. It has been shown that at $\rho=15 \mathrm{~dB}$, MSE of MMSE for ULA is higher in comparison with UCA when the central AOA is smaller than $50^{\circ}$. When central AOA becomes larger than $50^{\circ}$, the ULA performance is better in terms of lower value of MSE. For $\rho$ of $20 \mathrm{~dB}$ a similar trend has been observed but the cross point occurs for the central AOA equal to $60^{\circ}$. When the number of receiving antennas increases, the performance gets worse in terms of MSE for both ULA and UCA cases.

\section{References}

[1] J. Luo, J. R. Zeidler, and S. McLaughlin, "Performance analysis of compact antenna arrays with MRC in correlated Nakagami fading channels," IEEE Transactions on Vehicular Technology, Vol. 50, No. 1, January 2001.

[2] X. Liu, M. E. Bialkowski, and F. Wang, "Investigations into the effect of spatial correlation on channel estimation and capacity of multiple input multiple output system," International Journal of Communications, Network and System Sciences, Vol. 2, No. 3, June 2009.

[3] J. Tsai, R. M. Buehrer, and B. D. Woerner, "Spatial fading correlation function of circular antenna arrays with Laplacian energy distribution," IEEE Communications Letters, Vol. 6, No. 5, pp. 178-180, May 2002.

[4] J. Tsai, R. M. Buehrer, and B. D. Woerner, "The impact of AOA energy distribution on the spatial fading correlation of linear antenna array," Vol. 2, pp. 933-937, IEEE 5th VTC, May 2002. 
[5] E. G. Larsson and P. Stoica, "Space-time block coding for wireless communication," Cambridge University Press, 2003.

[6] C. N. Chuah, D. N. C. Tse, and J. M. Kahn, "Capacity scaling in MIMO wireless systems under correlated fading," IEEE Transactions on Information Theory, Vol. 48, pp. 637-650, March 2002.

[7] W. C. Jakes, Microwave Mobile Communications, John Wiley \& Sons, New York, 1974.

[8] J. G. Proakis, Digital Communications, 3rd Edition, McGraw-Hills, New York, 1995.

[9] M. Simon and M. S. Alouini, "A unified approach to the performance analysis of digital communication over generalized fading channels," Proceedings of IEEE, pp. 1860-1877, September 1998.

[10] E. Telatar, "Capacity of multi-antenna Gaussian channels," Europe Transactions on Telecommunication, Vol. 10, No. 6, pp. 585-596, November 1999.

[11] T. L. Marzetta and B. M. Hochwald, "Capacity of a mobile multiple-antenna communication link in Rayleigh flat fading," IEEE Transactions on Information Theory, Vol. 45, No. 1, pp. 139-157, January 1999.
[12] D. Shiu, J. Foschini, M. J. Gans, and J. M. Kahn, "Fading correlation and its effect on the capacity of multielemnt antenna system," IEEE Transactions on Communications, Vol. 48, No. 3, March 2000.

[13] M. Biguesh and A. B. Gershman, "MIMO channel estimation: Optimal training and tradeoffs between estimation techniques," Proceedings of ICC'04, Paris, France, June 2004.

[14] M. Biguesh and A. B. Gershman, "Training-based MIMO channel estimation: A study of estimator tradeoffs and optimal training signals," IEEE Transactions on Signal Processing, Vol. 54, No. 3, March 2006.

[15] X. Liu, M. E. Bialkowski, and S. Lu, "Investigations into training-based MIMO channel estimation for spatial correlated channels," Proceedings of IEEE AP-S Symposium, Hawaii, USA, 2007.

[16] X. Liu, S. Lu, M. E. Bialkowski, and H.T. Hui, "MMSE channel estimation for MIMO system with receiver equipped with a circular array antenna," Proceedings of 2007 Asia Pacific Microwave Conference, APMC2007, pp. 1-4, December 2007. 